\title{
The Ongoing Battle for Syringe Exchange
}

\author{
Baker A
}

Carlow University, Pennsylvania, United States

*Corresponding author: Baker A, Carlow University, Pennsylvania, United States, E-mail: bakera496@gmail. com

Citation: Baker A (2016) The Ongoing Battle for Syringe Exchange. J Aids Hiv Infec 2(1): 101. doi:

10.15744/2454-499X.2.101

Received Date: April 01, 2016 Accepted Date: July 05, 2016 Published Date: July 07, 2016

\begin{abstract}
Syringe exchange programs (SEPs) have been shown to be effective in reduction of infectious disease rates in several studies over the years. However, widespread implementation and use has proven to be a challenge. This article is an editorial discussing the benefits of syringe exchange programs and potential reasons behind the barriers associated with their implementation.
\end{abstract}

Abbreviations: PWID: Persons Who Inject Drugs; SEPs: Syringe Exchange Programs; HIV: Human immunodeficiency virus; HCV: Hepatitis C virus; WHO: World Health Organization

Intravenous drug use is a direct route of transmission of both hepatitis $\mathrm{C}$ and HIV. The most recent surveys of active intravenous drug users indicate that approximately one third of those ages 18-30 are infected with HCV, and older or former intravenous drug users typically have a drastically higher prevalence [1]. According to the World Health Organization, approximately 16 million people inject drugs worldwide, and 3 million of them are HIV positive [2]. In 2010, 8\% of new HIV infections in the US were attributed to injection drug use [3]. In 2009, PA had the $6^{\text {th }}$ highest number of all 50 states of newly diagnosed HIV cases, with 1736 new cases and an estimated lifetime treatment cost of $\$ 637$ million. California, Florida, New York, Texas and Georgia all had even higher numbers, and the total lifetime treatment cost for HIV based on new diagnoses in 2009 in the US was estimated to be $\$ 16.6$ billion [4]. In addition, according to the CDC (2016), in one study, $65 \%$ of IV drug users with HIV reported being homeless, and $44 \%$ reported having no health insurance in the last 12 months, therefore making it difficult for them to seek treatment [3].

Due to the high prevalence of HIV and hepatitis in the intravenous drug using population, it is imperative that measures be taken to prevent the spread of infection. One such strategy is the implementation of syringe exchange programs (SEPs), whereby persons who inject drugs (PWID) can access sterile needles and syringes free of cost. Although several studies have demonstrated their effectiveness, they have not been widely accepted in the US, and are not being used to their full potential. As previously mentioned, PA was $6^{\text {th }}$ highest in terms of numbers of newly diagnosed HIV cases in 2009 . Considering that six out of 50 is pretty significant, one would think that public officials would be putting in efforts to reduce these numbers. However, in the entire state, syringe exchange is only available in Pittsburgh and Philadelphia, and the hours of operation are extremely limited. Syringe exchange not only provides clean needles and equipment to PWID, but also provides a place for disposal of used equipment. That in itself is undervalued. Without a place to properly dispose of used needles that are potentially infected, PWID are left to either throw them in the trash or on the street, which places the rest of the population at risk of accidentally coming in contact with and being stuck by a contaminated needle. Furthermore, it has been shown numerous times through a variety of study designs that SEPs reduce the spread of infection among users. In addition, most sites not only provide syringes and equipment, but they are a gateway to other invaluable services. For example, Prevention Point is an organization that provides syringe exchange in both Philadelphia and Pittsburgh. Not only do they provide sterile equipment, but they offer a number of other services including HIV and Hepatitis $\mathrm{C}$ testing, case management, crisis intervention and counseling, overdose prevention and response education with Narcan prescription, assistance to drug treatment, healthcare, housing, food pantries, and other social services. These programs also offer a safe, nonjudgmental place where PWID who are often stigmatized can seek help that goes beyond substance use. Researchers in Canada conducted a small qualitative study across four SEP sites, and concluded that most clients indicated that drug use was a way of numbing both physical and emotional pain [5]. The SEP sites were unanimously described as a safe haven in an often unsafe world by all participants [5].

In regards to the impact of injection drug use on HIV and HCV rates, epidemiologic data has been difficult to obtain. However, according to WHO, one out of every ten new HIV infections is caused by injecting drug use and in parts of Eastern Europe and Central Asia over 80 per cent of all HIV infections is related to drug use [2]. In addition, according to Klevens, et al. (2012), National viral hepatitis surveillance from 2009 confirmed that 241 of 432 acute HCV cases who responded to the question, "Have you used injection drugs in the past 6 months?" answered "yes." [6]. 
If this reasoning isn't enough to convince those in opposition, then cost effectiveness might be. The costs of HCV infection and HIV are extremely high, even with the use of antivirals. Today, the total estimated cost of HCV infection is $\$ 6.5$ (\$4.3-\$8.4) billion and it expected to peak in 2024 at $\$ 9.1$ (\$6.4-\$13.3) billion [7]. Furthermore, a study conducted in China examining the cost effectiveness of SEPs concluded that spending of a total USD $\$ 1.04$ million on NSPs from 2002 to 2008 has resulted in cost-savings of USD\$1.38-1.97 million due to the prevention of HIV and the associated costs of care and management [8]. Many PWID do not have access to healthcare and therefore, without programs like SEPs, they may go undiagnosed and therefore untreated for extended periods of time, often seeking treatment once the disease has already advanced, further increasing costs of care.

Considering all the benefits, what is the problem? In 2012, the Consolidated Appropriates Act prohibited the use of federal funds for the distribution of needles or syringes for injection of any illegal drug [9]. This act specifically banned the use of federal funds for human resources used specifically to distribute needles and syringes; purchases of needles or syringes; or delivery modes such as vehicles or rent for fixed sites used specifically for the distribution of needles and syringes [9]. However, in December of 2015, the Consolidated Appropriations act of 2016 was signed into law, and does give local communities and states the opportunity to use federal funds to support certain components of SEPs [10]. While this is a step in the right direction, the law does require consulting with the CDC initially in order to demonstrate the need for the program, and then working with federal funding agencies to request such funds [10].

Funding is only one of several barriers to achieving the potential outcomes of SSPs. The stigma associated with injection drug use may inhibit many users from seeking help. Fear of legal consequences is also a deterring factor. It has been shown that police interference has a negative impact on the success of SEPs. Syringe confiscation, uninvited appearances at SEPs, use of SEP membership as a criminality and client arrest have also been issues [11]. Both experience with and perception of negative police encounters can decrease willingness to participate in SEPs and other risk reduction practices [11]. In a cross sectional study including a national survey of SEPs aimed at determining associations between policing interference and characteristics of US SEPs, Beletsky, et al. (2010), found that client harassment by police was the most frequent adverse outcome reported, and that the second most frequent was unauthorized confiscation of clients' injection equipment [11]. Other adverse events included client arrest en route to or from the exchange. Clearly, this would be a major barrier to client use of the programs. Of note, the authors of this study also found that there was a greater risk of client arrest and unauthorized confiscation of injection equipment reported by SEPs serving primarily people of color [11]. This has huge consequences considering the fact that this could deter this population from using the SEPs even mores, ultimately contributing to increased incidence of disease in minority communities [11]. Interestingly, the authors also concluded that sites that were more visible, serving more sites per week, were more likely to experience police interference [11]. This has huge implications. Limited access to services is a barrier on its own. However, if increasing access also increases police interference, then it's a catch 22 . In my opinion, this clearly demonstrates the need for community wide education for police. Law enforcement, community groups, and public health officials need to work together to overcome this barrier. It may take a great deal of effort, and some compromise from both sides, but it is absolutely necessary to ensure that these occurrences do not continue. How is it that a PWID can legally go to a SEP and receive equipment, and then have it confiscated on their way home? There is clearly a major problem that needs to be resolved sooner rather than later.

Despite the known benefits of SEPs, we have not come very far in their implementation over the years. The World Health organization recommends providing 200 sterile syringes per drug injector per year in order to effectively reduce HIV transmission via this route. However, the most recent estimates from 2010 found that only approximately 23 syringes were distributed per PWID per year [12]. This statistic in itself sums up the amount of work that needs to be done to improve this issue. The recent lift on the ban for federal funding may be the first step in successful implementation of SEPs, but it is up to health officials, law enforcement and the community as a whole to contribute to the acceptance of these programs, which will overall contribute to their success and improve the health of our country.

\section{References}

1. CDC (2016) Hepatitis C FAQs for Health Professionals.

2. WHO (2016) HIV/AIDS: Injecting Drug Use.

3. CDC (2015) HIV and injection drug use in the United States.

4. CDC (2105) HIV Cost effectiveness.

5. Macneil J, Pauly B (2011) Needle exchange as a safe haven in an unsafe world. Drug and Alcohol Review 30: 26-32.

6. Klevens RM, Hu DJ, Jiles R, Holmberg SD (2012) Evolving epidemiology of hepatitis C virus in the United States. Clin Infect Dis s3-9.

7. Razavi H, Elkhoury AC, Elbasha E, Estes C, Pasini K, et al. (2013) Chronic hepatitis C virus (HCV) disease burden and the cost in the United States. Hepatology 57: 2164-70.

8. Zhang L, Yap L, Xun A, Wu Z, Wilson D (2011) Needle and syringe programs in Yunnan, China yield health and financial return. Biomed Central Public Health DOI: $10.1186 / 1471-2458-11-250$.

9. CDC (2015) Access to sterile syringes.

10. CDC (2016) Access to sterile syringes.

11. Beletsky L, Grau LE, White E, Bowman S, Heimer R (2010) The roles of law, client race and program visibility in shaping police interference with the operation of US syringe exchange programs. Addiction 106: 357-65. 
12. AVERT (2015) Needle and syringe programmes for HIV prevention.

Submit your next manuscript to Annex Publishers and
benefit from:
$>$ Easy online submission process
$>$ Online article availability soon after acceptance for Publication
$>$ Open access: articles available free online
Submit your manuscript at
http://www.annexpublishers.com/paper-submission.php 